\title{
Scar wars
}

\section{W. J. Ferguson, Professor at School of Biological Sciences, University of Manchester}

Scarring following trauma, injury, burns or surgery causes serious medical, psychological and functional problems, for which there are currently no good preventative or therapeutic treatments.

Most burn injuries occur in children under the age of 16 and can result in life long surgical and psychological therapies to counteract the adverse sequelae of scarring. Equally in cleft lip repair contraction of the scar tissue collagen often results in adverse growth of the mid face and consequential surgical/orthodontic therapy.

Scarring is not just a problem in the skin: it occurs in all body tissues and organs. Thus for example, scarring in the eye following a penetrating injury or laser surgery can lead to cloudy vision, or in the worst case, blindness; glial scarring in the brain following neurosurgery provides a physical barrier to nerve reconnection, whilst scarring in the intestinal and reproductive organs often leads to adhesions

developing and so the numbers and differentiation status of inflammatory cells recruited to the embryonic wound site is very different from that in the adult. As a consequence the growth factor profile at the embryonic wound site is different from that in the adult.

We utilised this information to experimentally manipulate adult wound healing. We reduced the levels of growth factors present at high levels in the adult, but low levels in the embryo, e.g. Transforming Growth Factor Beta 1 and Beta 2 (TGFb1 and TGFb2) or elevated levels of growth factors present a high levels in an embryonic wound, but low levels in the adult wound (TGFb3). These experimental manipulations resulted in adult wounds

\section{We became interested in the biology of scarring many years ago when we observed that wounds made on early embryos. . healed perfectly with no scar.}

and strictures, which can be life threatening. Equally, the cellular and molecular mechanisms underlying scar tissue formation are also shared by a number of fibrotic disorders e.g, liver cirrhosis, pulmonary fibrosis, glomerulonephritis, for which there are currently no good therapies.

We became interested in the biology of scarring many years ago when we observed that wounds made on early embryos (in the course of cleft lip and palate research) healed perfectly with no scar. We investigated the cellular and molecular basis of this scar free embryonic healing and contrasted it with mechanisms in the adult using a variety of experimental models.

There are many differences between embryonic and adult healing, but importantly for scar formation, the inflammatory response in the embryo is different, both quantitavely and qualitatively.

The immune system in an embryo is that healed perfectly with no signs of scar formation. We utilised transgenic technology to manipulate the levels of endogenous gene expression of these important growth factors in embryonic and adult wounds in order to verify the importance of these molecules in scar formation.

Following on from the results of these extremely encouraging pre-clinical experiments we have commenced development of these agents as potential human therapeutics. This has been conducted by a University Biotechnology spin-out company, Renovo Limited (www.renovo.com). We have conducted appropriate safety and toxicology studies and following satisfactory results for the drug candidate, have manufactured clinical grade pharmaceuticals, which are now in human clinical trial. Early results look promising.

Such scar preventing therapies could find widespread medical/surgical usage, e.g., following burn injury, major trauma e.g road traffic accident, all forms of elective surgery, cosmetic surgery, scar revision surgery etc.

Moreover, many surgical operations, particularly on the face, place the incision site in cosmetically acceptable areas and then use extensive manipulations and special equipment to access the area of interest. If scar free healing could be assured in a majority of patients, then direct incisions over the site and simpler, faster surgery may be possible. Moreover, many individuals may wish to get rid of existing scars: our therapies could offer potential, e.g., scar revision surgery, combined with application of the scar preventing medicines.

In this context it is of interest to note that a recent UK Psychological Survey estimated that $67 \%$ of all women in the UK had a psychological problem with a scar: often only a scar visible on intimate contact, e.g. from a past caesarean section or hysterectomy.

Finally wounds in certain areas of the oral cavity e.g., gingival papillae, can heal with perfect regeneration and share many of the cellular and molecular features of scar free embryonic healing. Such findings are not surprising given the repeated daily microtrauma in the oral cavity from eating, speaking and other forms of oral function. Maybe in the future we can extend the special healing powers of the mouth to other areas of the body with advantageous medical, surgical and psychological outcomes!

The 2002 British and Irish Dental Associations's Annual Conference is being held at Belfast Waterfront Hall between 2-4 May 2002.

Contact BDA Events office for further information. Tel: +44 (020) 7563 4590; Fax: +44 (020) 75634591.

Website: www.bda-events.org.uk 